\title{
Beam Forming Algorithm Based On Symmetric Alpha-stable Distribution
}

\author{
Peng Cheng \\ Electronic Engineering College \\ Navy University of Engineering \\ Wuhan, China \\ E-mail: 992046452@qq.com
}

\author{
Wang Ping Bo \\ Electronic Engineering College \\ Navy University of Engineering \\ Wuhan, China \\ E-mail: 18672325415@163.com
}

\author{
Jiang Jin Cun \\ Electronic Engineering College \\ Navy University of Engineering \\ Wuhan, China \\ E-mail: 992046452@qq.com \\ Zhang Peng Kun \\ Electronic Engineering College \\ Navy University of Engineering \\ Wuhan, China \\ E-mail: 18672325415@163.com
}

\begin{abstract}
Via modeling the reverberation noise by means of the symmetric $\alpha$-stable $(\alpha S)$ distribution, and the secondorder and above order moments of $\alpha S$ distribution are nonexistent, the fractional lower-order moment and zeroorder moment concepts are put forward and the beam forming algorithms on account of them are proposed to instead of the beam forming algorithms on account of second-order moments. Taken full advantage of the feature of no second-order or above order existing in the symmetrical $\alpha$-stable( $S \alpha S)$ process, the reverberation noise which possesses pulse characteristic can be strongly inhibited by these two algorithms, resulting in overcoming the shortage that the traditional beam former on the basis of second-order statistical moment was not suitable for reverberation noise. Compared with the traditional ones, more efficient algorithm performance and better robustness are showed out by the experiments. And the experimental results which based on sea -test data also show that these two algorithms are very efficient.
\end{abstract}

Keywords-Symmetric; a-Stable Dsitribution; Beam Forming; Fractional Lower-order Moment; reverberation; Sonar

\section{INTRODUCTION}

Beam forming technology is one of the effective tools in modern array signal processing procedure. In essence, beamforming can be considered as a spatial filtering wave, which is used to weight the array receiving data based on some kind of criterion [1], to enhance the desired signal and suppress interference and noise and its performance is decided by the choice of the beamforming weight vector. Usually the noise and interference in the received array signal are assumed to conform with the Gauss distribution in traditional signal processing, and adopting the methods based on second-order statistics (variance, correlation, covariance) for further analysis and treatment is very effective in most cases. However, in some fields such as radar, communication and sonar and so on, many interference and noise do not obey the Gauss distribution. For instance, there are amplitude spikes existing in those signals including atmospheric lightning noise, underwater reverberation, instantaneous spike on the telephone lines and their probability densities have algebraic tails $[2,3,13]$. Studies have shown that the $S \alpha S$ process is more suitable to describe the reverberation signal of underwater noise [4,14], so distribution is used to describe active sonar reverberation in this paper, with the statistical moments without beyond the scope of $\alpha(0<\alpha \leq 2) \quad[2,3,15]$. Traditional adaptive beamforming algorithm based on second-order can not acquire satisfactory results, and even leads to the performance degradation. Therefore, in reverberation interference environment, traditional methods must be improved through using fractional lowerorder statistical moments to replace second-order statistical moments.

$\alpha$-stable distribution has no unified and closed probability density function expression [2,3,16], but its characteristic function is existing. So we usually use the characteristic functions under the standard parameters system to describe it:

$$
\varphi(t)= \begin{cases}\exp \left\{-\sigma^{\alpha}|t|^{\alpha}[1-j \beta(\operatorname{sign}(t))] \tan \left(\frac{\pi \alpha}{2}\right)+\mathrm{j} \mu \mathrm{t}\right\}, & \alpha \neq 1 \\ \exp \left\{-\sigma|t|\left[1+j \beta(\operatorname{sign}(t)) \frac{2}{\pi} \log |t|\right]+j \mu t\right\}, & \alpha=1\end{cases}
$$

(1)

Where

$$
\operatorname{sign}(t)=\left\{\begin{array}{ll}
1, & t>1 \\
0, & t=1 \\
-1, & t<1
\end{array} \quad, \quad \gamma=\sigma^{\alpha}\right.
$$

$\alpha$ is called characteristic exponent, $\beta$ is deviation index, $\gamma$ is dispersion coefficient, and $\mu$ is location parameter, characteristic exponent $\alpha$ is the most important parameter. And it is Gauss distribution when $\alpha=2$. So we 
consider that the Gauss distribution is a special case of stable distribution. Levy presented the $\alpha$-stable distribution for the first time when he researched the generalized central limit theorem in $1925[5,6]$ and pointed out that it was the only limiting distribution of the sum of the independent identically distributed random variables. Compared with the Gauss distribution, it has a wider range of applicability.

\section{BEAMFORMING}

Based on the array element geometry, sonar array can be divided into three categories: linear array, planar array and volumetric array. Linear array and planar array can be considered as special cases of volumetric array. Linear array is the most commonly used form of matrix array, and a kind of the easiest ways to be analyzed. Linear array is divided into non uniform linear array and uniform linear array dominanting the means of sonar signal receiving. Each desired signal of array element arriving at weight matrix corresponding to the delay sample transporting through different distances carried through reverse delay compensation sum to improve the output signal-to-noise ratio, which is called Conventional Beam Forming(CBF). In general, the underwater acoustic signal is satisfy the far field conditions, namely the active sonar echo signal or underwater target signal approximation is thought to parallel to the plane wave. Array model is shown in Fig .1.

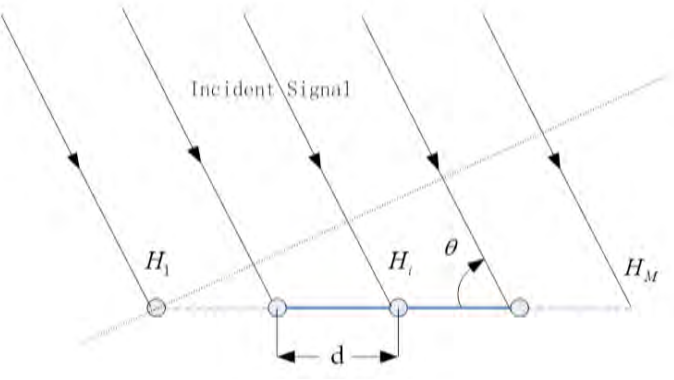

Figure 1. Uniform linear array model

Using array element $H_{1}$ as the reference, the received array signal of the array element $i$ can be approximated by:

(3)

$$
s_{i}(t)=s(t) e^{-j \omega \tau_{i}}=s(t) e^{-j \frac{2 \pi(i-1) d \cos \theta}{\lambda}}
$$

In the actual sonar array, a desired signal and $K-1$ interference signals incident respectively from $\theta_{1}, \theta_{2}, \cdots, \theta_{\kappa}$, and direction vectors of the $K$ sources are expressed as $\mathbf{a}\left(\theta_{1}\right), \mathbf{a}\left(\theta_{2}\right), \cdots, \mathbf{a}\left(\theta_{\kappa}\right)$. So the array manifold matrix can be approximated by $A=\left[\mathbf{a}\left(\theta_{1}\right), \mathbf{a}\left(\theta_{2}\right), \cdots, \mathbf{a}\left(\theta_{K}\right)\right]$ [7]. The snapshot sampling value of the nth snapshot expressed by [8]:

(4)

$$
\mathbf{x}\left(t_{n}\right)=\mathbf{A S}\left(t_{n}\right)+\mathbf{n}\left(t_{n}\right)
$$

Where

$$
\mathbf{a}\left(\theta_{k}\right)=\left[1, e^{-\frac{2 \pi d \cos \theta_{i}}{\lambda}}, \mathrm{L}, e^{-\frac{2 \pi(M-1) d \cos \theta_{i}}{\lambda}}\right]^{\mathrm{T}}
$$

$$
\mathbf{S}\left(t_{n}\right)=\left[S_{1}\left(t_{n}\right), S_{2}\left(t_{n}\right), \mathrm{L}, S_{K}\left(t_{n}\right)\right]^{\mathrm{T}}
$$

$$
\mathbf{n}\left(t_{n}\right)=\left[n_{1}\left(t_{n}\right), n_{2}\left(t_{n}\right), \mathrm{L}, n_{M}\left(t_{n}\right)\right]^{\mathrm{T}}
$$

Self-adaptive beamforming is exactly to use snapshot data of each array element to derive the beamforming weight vector. The output signal of uniform linear array is expressed as:

$$
y(t)=\mathbf{w}^{\mathrm{H}} \mathbf{x}(t)
$$

Where, $\mathbf{w}=\left[w_{1}, w_{2}, \mathrm{~L}, w_{w}\right]$ is beamforming weight vector, and the beam response is formulated as follows:

$$
B\left(\theta, \theta_{0}\right)=\mathbf{w}^{\mathrm{H}} \mathbf{a}(\theta), \quad \theta \in\left[0^{\circ}, 180^{\circ}\right]
$$

\section{FRMVDR BEAMFORMING ALGORITHM}

Reverberation noise signal obeys $S \alpha S$ distribution [9], with an unbounded second-order moment leading to traditional beamforming algorithm inapplicable. So we use fractional lower-order moment instead of second-order moment. The conjugate $\mathrm{p}$ norm of $\mathrm{x}$ is defined as follows:

$$
x^{\langle p\rangle}=|x|^{p-1} x, \quad 0 \leq p \leq 1
$$

For the faculative of $\mathrm{M}$-dimensional vector $\mathrm{x}$, the conjugate $\mathrm{p}$ norm is as follows:

$$
\mathbf{x}^{\langle p\rangle}=\left[x_{1}^{\langle p\rangle}, x_{2}^{\langle p\rangle}, \mathrm{L}, x_{M}^{\langle p\rangle}\right]^{\mathrm{T}}
$$

The array response of moment $t_{n}$ is defined:

$$
y\left(t_{n}\right)=\sum_{i=1}^{M} w_{i}{ }_{i} x_{i}^{\langle p\rangle}\left(t_{n}\right)=\mathbf{w}^{\mathrm{H}} \mathbf{x}^{\langle p\rangle}\left(t_{n}\right)
$$

Where, $*$ means to obtain the complex conjugate, $H$ means to obtain the conjugate transpose. The above formula called fractional lower order array response. The output power of fractional lower order array response is defined as:

(13)

$$
P(\mathbf{w})=E\left\{|y(t)|^{2}\right\}=\mathbf{w}^{\mathrm{H}} E\left\{\mathbf{x}^{\langle p\rangle}(t)\left[\mathbf{x}^{\langle p\rangle}(t)\right]^{\mathrm{H}}\right\} \mathbf{w}=\mathbf{w}^{\mathrm{H}} \mathbf{R}^{\langle p\rangle} \mathbf{w}
$$

Where

$$
\mathbf{R}^{\langle p\rangle}=E\left\{\mathbf{x}^{\langle p\rangle}(t)\left[\mathbf{x}^{\langle p\rangle}(t)\right]^{\mathrm{H}}\right\}
$$

When $p=1$, so $\mathbf{R}=E\left\{\mathbf{x}(t) \mathbf{x}^{\mathrm{H}}(t)\right\}$ is traditional covariance matrix, the algorithm degenerates into Minimum Variance Distortlessness Response(MVDR)[10]. Since $S \alpha S$ distribution does not possess finite second-order moments, so $\mathbf{R}^{\langle p\rangle}$ is bounded, if we take $p<\alpha / 2$ [7,11]. MVDR algorithm with preprocessing of the conjugate $\mathrm{p}$ norm to random various $\mathrm{x}$, is called Fractional Minimum Variance Distortlessness Response(FrMVDR). FrMVDR beamformer is formulated as follows: 


$$
\left\{\begin{array}{l}
\underset{{ }^{*}}{\min } E\left\{|y(t)|^{2}\right\} \\
\text { s.t. }{ }^{\mathrm{H}} \mathbf{a}\left(\theta_{1}\right)=1 \\
\mathbf{x}^{\langle p\rangle}(t)=\left[x_{1}^{\langle p\rangle}(t), x_{2}^{\langle p\rangle}(t), \mathrm{L}, x_{M}^{\langle p\rangle}(t)\right]
\end{array}\right.
$$

The physical meaning contained above is: in the case of ensuring the desired signal without distortion, the output power of fractional lower order array beamformer minimum. The optimal weight vector solving methods of FrMVDR and MVDR are alike, for that both methods use the Lagrange vector factor as solving approach. The theory of optimal weight vector in the desired direction is:

$$
\mathbf{W}_{\text {opt }}=\frac{\left[\mathbf{R}^{\langle p\rangle}\right]^{-1} \mathbf{a}\left(\theta_{0}\right)}{\mathbf{a}^{\mathrm{H}}\left(\theta_{0}\right)\left[\mathbf{R}^{\langle p\rangle}\right]^{-1} \mathbf{a}\left(\theta_{0}\right)}
$$

In practical application, we use $\mathrm{N}$ sampling values in different time of the array to estimate matrix $\mathbf{R}^{\langle p\rangle}$ :

$$
\hat{\mathbf{R}}^{\langle p\rangle}=\frac{1}{N} \sum_{n=1}^{N} \mathbf{x}^{\langle p\rangle}\left(t_{n}\right)\left[\mathbf{x}^{\langle p\rangle}\left(t_{n}\right)\right]^{\mathrm{H}}
$$

Where, the value of $\mathrm{p}$ must be pre-determined, so the priori knowledge of characteristic exponent $\alpha$ need to be aware [12], selecting a suitable parameter value for $\mathrm{p}$ from $[0, \alpha / 2]$. At this point the output power and optimal weight vector of receive array signal are as follows:

$$
E\left\{|y(t)|^{2}\right\}=\frac{1}{N} \sum_{n=1}^{N}\left|y\left(t_{n}\right)\right|^{2}=\hat{\mathbf{w}}_{\text {op }}^{H} \hat{\mathbf{R}}^{\langle p\rangle} \hat{\mathbf{w}}_{\text {opt }}
$$

$$
\hat{\mathbf{W}}_{\mathrm{opt}}=\frac{\left[\hat{\mathbf{R}}^{\langle p\rangle}\right]^{-1} \mathbf{a}\left(\theta_{0}\right)}{\mathbf{a}^{\mathrm{H}}\left(\theta_{0}\right)\left[\hat{\mathbf{R}}^{\langle p\rangle}\right]^{-1} \mathbf{a}\left(\theta_{0}\right)}
$$

\section{ZMVDR BEAMFORMING ALGORITHM}

The application of FrMVDR beamforming algorithm is limited to the selection of value of parameter $p$, so we present a zero order array beam response algorithm, namely Zero Minimum Variance Distortlessness Response (ZMVDR), to overcome this drawback of FrMVDR algorithm. The complex random variable $x=r e^{j \theta}$ is represented by the polar coordinate form, so $\ln x=\ln r+j \theta$, and zero norm of $x$ is defined as:

$$
x^{\langle 0\rangle}=\operatorname{real}(\ln x) e^{j \operatorname{mimag}(\ln x)}=(\ln r) e^{j \theta}
$$

Zero norm of $\mathrm{M}$ dimensional vector is defined as follows:

$$
\mathbf{x}^{\langle 0\rangle}=\left[x_{1}^{\langle 0\rangle}, x_{2}^{\langle 0\rangle}, \mathrm{L}, x_{M}^{\langle 0\rangle}\right]^{\mathrm{T}}
$$

Formula (19) shows that $\mathbf{x}^{\langle 0\rangle}$ only changes the amplitude information of $\mathbf{x}$, without changing its phase information. Array response of time $t_{n}$ is expressed as follows:
We call (22) Zero-order array response. The zero-order array output power is expressed as follows:

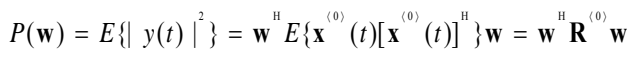

For the purposes of a finite number of sampling points, matrix $\mathbf{R}^{\langle 0\rangle}$ is always bounded, so output power is present. ZMVDR algorithm can be expressed as:

$$
\left\{\begin{array}{l}
\underset{\mathbf{w}}{\min } E\left\{|y(t)|^{2}\right\} \\
\text { s.t. }{ }^{\mathrm{H}} \mathbf{a}\left(\theta_{1}\right)=1 \\
\mathbf{x}^{\langle 0\rangle}(t)=\left[x_{1}^{\langle 0\rangle}(t), x_{2}^{\langle 0\rangle}(t), \mathrm{L}, x_{M}{ }^{\langle 0\rangle}(t)\right]
\end{array}\right.
$$

The physical meanings of ZMVDR and FrMVDR are similar. The optimal weight vector solving methods of ZMVDR and MVDR are alike, for that both methods use the Lagrange vector factor as solving approach. The theory of optimal weight vector in the desired direction is:

$$
\mathbf{w}_{\text {opt }}=\frac{\left[\mathbf{R}^{\langle 0\rangle}\right]^{-1} \mathbf{a}\left(\theta_{0}\right)}{\mathbf{a}^{\mathrm{H}}\left(\theta_{0}\right)\left[\mathbf{R}^{\langle 0\rangle}\right]^{-1} \mathbf{a}\left(\theta_{0}\right)}
$$

In practical application, we use $\mathrm{N}$ sampling values in different time of the array to estimate matrix $\mathbf{R}^{\langle 0\rangle}$ :

$$
\hat{\mathbf{R}}^{\langle 0\rangle}=\frac{1}{N} \sum_{n=1}^{N} \mathbf{x}^{\langle 0\rangle}\left(t_{n}\right)\left[\mathbf{x}^{\langle 0\rangle}\left(t_{n}\right)\right]^{\mathrm{H}}
$$

The output power and optimal weight vector of receive array signal are following:

$$
\begin{aligned}
E\left\{|y(t)|^{2}\right\} & =\frac{1}{N} \sum_{n=1}^{N}\left|y\left(t_{n}\right)\right|^{2}=\hat{\mathbf{w}}_{\mathrm{opt}}^{\mathrm{H}} \hat{\mathbf{R}}^{\langle 0\rangle} \hat{\mathbf{w}}_{\mathrm{opt}} \\
\hat{\mathbf{w}}_{\mathrm{opt}} & =\frac{\left[\hat{\mathbf{R}}^{\langle 0\rangle}\right]^{-1} \mathbf{a}\left(\theta_{0}\right)}{\mathbf{a}^{\mathrm{H}}\left(\theta_{0}\right)\left[\hat{\mathbf{R}}^{\langle 0\rangle}\right]^{-1} \mathbf{a}\left(\theta_{0}\right)}
\end{aligned}
$$

\section{EXPERIMENTAL RESULTS}

In order to verify the performance of the above beamforming algorithms and compare with traditional method, we processed the sea test data. The data came from [0601-715 sanya sea test]. Transmitting signal was Linear Frequency Modulaton(HMF) pulse with frequency ranging from $1.5 \mathrm{kHz}$ to $2.0 \mathrm{kHz}, 4$ seconds pulse width, target azimuth $\theta_{0}=116^{\circ}$. We used 96 uniform linear arrays with $d=0.45$ meter element spacing to receive signals, along with $6.0 \mathrm{kHz}$ sampling rate. Datas of the first 16 array elements were collected to input into the beamformer, following with pretreatment via $600 \mathrm{~Hz}$ intermediate frequency FIR bandpass filter of 512-order before beamforming. To make the calculation simple, here we only considered a subset of narrow-band emission signal center frequency: $f=1750 \mathrm{~Hz}$. The three beam graphes formed by beamformer are shown from Fig .2 to Fig .5. 


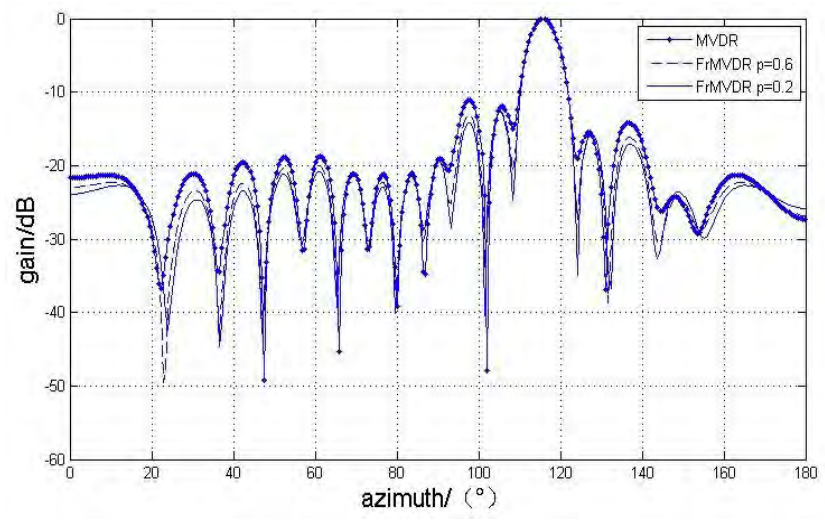

Figure 2. Beam graphes of MVDR and FrMVDR

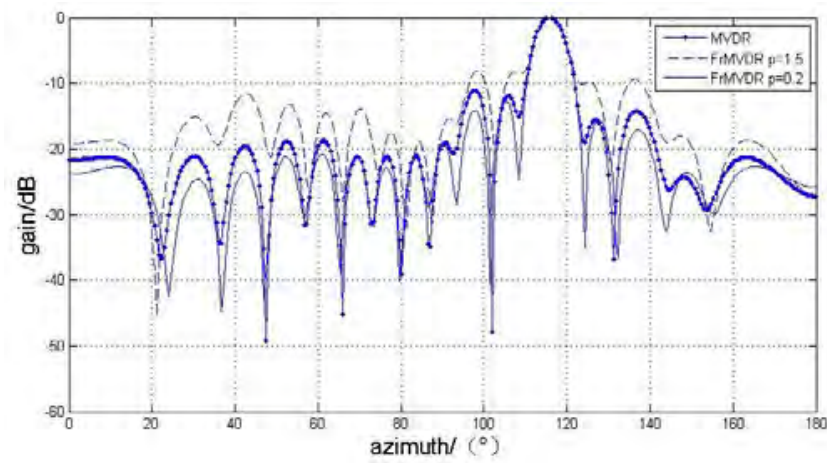

Figure 3. Distortion beam graphes of FrMVDR

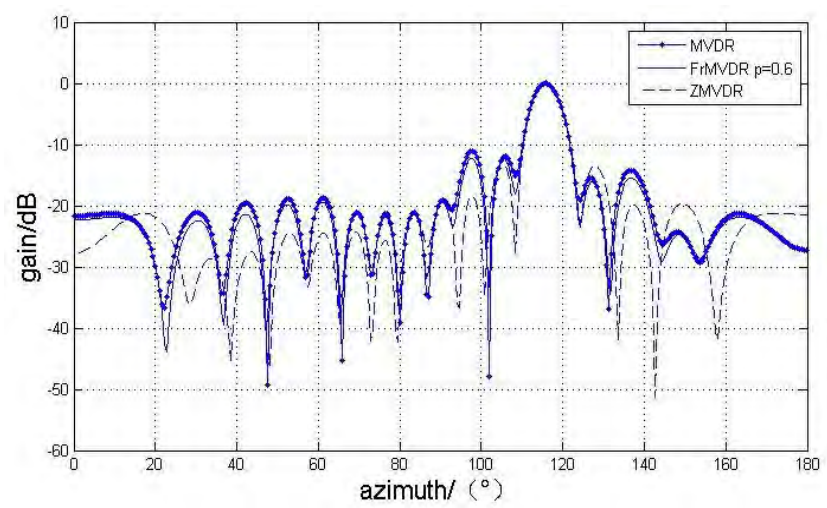

Figure 4. Beam graphes of MVDR,FrMVDR and ZMVDR $(\mathrm{p}=0.6)$

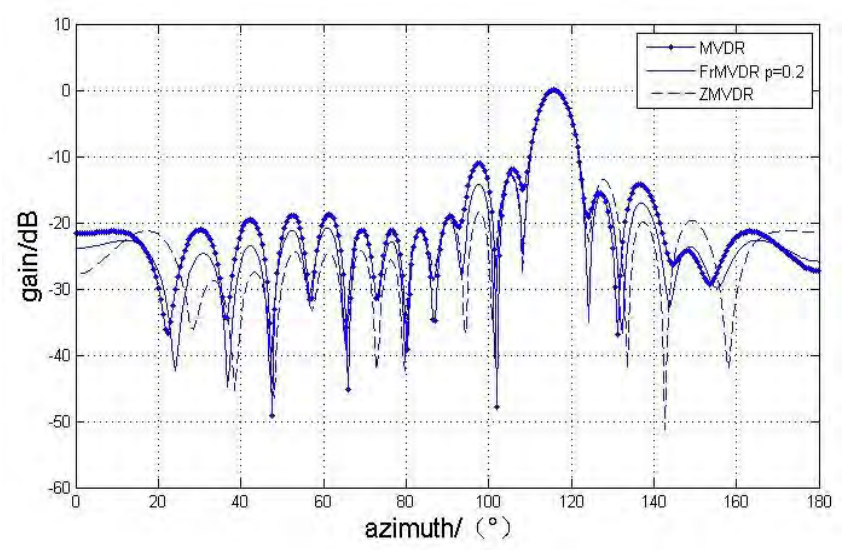

Figure 5. Beam graphes of MVDR,FrMVDR and ZMVDR $(\mathrm{p}=0.2)$
In the view of Fig 2 and Fig 4, MVDR, FrMVDR and ZMVDR beamforming algorithms can produce high gain in the direction of the desired signal. Compared with MVDR, FrMVDR and ZMVDR have better overall inhibitory effect on the sidelobe. Because parameter influenced the inhibitory effect of sidelobe in FrMVDR there would appear distorted when $p \geq \alpha / 2$, and FrMVDR algorithm became stronger on the whole sidelobe suppression while adopting a smaller $\mathrm{p}$ value when $p \geq \alpha / 2$. Although some secondary sidelobe inhibition of ZMVDR faded next to FrMVDR, ZMVDR is obviously more effective on adjacent null. Meanwhile ZMVDR proved to have stronger robustness due to have no inpendencies on the priors of $\alpha$.

\section{CONCLUSION}

The concepts of fractional lower order statistics and the zero order statistical moments are presented in this paper, following with deriving the beam forming algorithms of fractional lower order moments and zero order moments based on $S \alpha S$ distribution environment, and analysis the performance of beamformer. Because the active sonar reverberation noise signal obeys $S \alpha S$ distribution, and there is no p-order $(p>\alpha)$ moment existing, the traditional beamforming algorithm based on second-order will be not applicable, which is finally and successfully solved by, the two algorithms described in this article utilizing fractional lower order moment and zero-order moment to replace second moment. We improve the traditional MVDR beamforming algorithm into FrMVDR algorithm and ZMVDR algorithm by processing $\mathrm{p}$ norm and zero norm pretreatment of array received signal before sending into beamformer. Experimental results show that, Both of FrMVDR and ZMVDR can acquire a high gain in the desired signal direction, better and obvious overall sidelobe suppression effect than MVDR. Meanwhile, without recourse to the priori values of $\alpha$, ZMVDR is proved to be a highly robust beamforming algorithm. These two algorithms are both suitable for processing reverberation signal which obey $S \alpha S$ distribution resulting in great significance for active sonar antireverberation.

\section{ACKNOWLEDGMENT}

This research is partially supported by the project of National Natural Science Foundation of China with grant No.51109218.

\section{REFERENCES}

[1] Wang Yong Liang, Ding Qian Jun, Li Rong Feng, Adaptive Array Processing, 1st ed, Bei Jing: Tsinghua University press, 2009, pp 13-18.

[2] NIKIAS C L, SHAO M, Signal Processing with Alpha-Stable Distributions and Applications, 1st ed, New York: John Wiley \& Sons Inc, 1995, pp. 100-178.

[3] SHAO M, NIKIAS C L, "Signal Processing with Fractional Lower Order Moments: Stable Processes and Their Applications", Proceedings of IEEE, vol. 81, Jul 1993, pp. 986-1010.

[4] Wang Ping Bo, Active Sonar Signal Processing Technology Research of Non-Gauss. Wu Han: Navy Engineering University, 2006

[5] Feller W, An Introduction to Probability Theory and Its Applications. New York: John Wiley \& Sons Inc, 1971. 
[6] Breiman L, Probability Neading. MA: Addison Wesley, 1968.

[7] Ward J, Space-time Adaptive Processing for Airborne Radar. Tech. Rep: Lincoln Laboratory, 1994.

[8] Li Qi Hu, "Sonar Technology in Twenty-first Century", Application of Acoustic, vol 21, Jan 2002, pp. 13-18.

[9] Shao M, Nikias C L, "Signal Detection in Impulsive Noise Based on Stable Distributions", 1993 Conference Record of The TwentySeventh Asilomar Conference on Signals, Systems and Computers, vol 1,Oct 1993, pp. 218-222

[10] Capon J, "High Resolution Frequency-wave Number Spectrum Analysis", Proceedings of IEEE, vol 57, Nov 1969, pp. 1408-1418.

[11] He Jin, "Pulse Adaptive Beam Forming Method of Robust in Noisy Environment", Chinese Journal of Electronics, vol 3, Mar 2006, pp 464-468.
[12] Tsihrintzis G A, Nikas C L, "Fast Estimation of The Parameters of Alpha-stable Impulsive Interference", IEEE Trans on SP, vol 44 May 1996, pp. 1492-1503.

[13] Vanveen BD, Buckley KM., "Beamforming: A Versatile Approach to Spatial Filtering", Signal Processing Magazine of IEEE, vol 2 , 1988, pp. 4-24.

[14] Wang Han Qing, Wang Ping Bo, "The Underwater Acoustic Signal Adaptive Beam Forming Algorithm Based on The $S \alpha S$ Distribution", Ship\&Ocean Engineering, vol 41, Aug 2012, pp. 165-167.

[15] Li J, Stoica P, Robust Adptive Beamforming, 1st ed, New York: John Wiley \& Sons Inc, 2006, pp. 65-93.

[16] Li J, Stoica P, Wang Z, "Doubly Constrained Robust Capon Beamformer", Transactions on Signal Processing of IEEE, vol 52 , Aug 2004, pp. 2407-2423. 